\title{
Extinctions in the random replicator model
}

\author{
Viviane M. de Oliveira and J. F. Fontanari \\ Instituto de Física de São Carlos \\ Universidade de São Paulo \\ Caixa Postal 369 \\ 13560-970 São Carlos SP, Brazil
}

\begin{abstract}
The statistical properties of an ecosystem composed of species interacting via pairwise, random interactions and deterministic, concentration limiting self-interactions are studied analytically with tools of equilibrium statistical mechanics of disordered systems. Emphasis is given to the effects of externally induced extinction of a fixed fraction of species at the outset of the evolutionary process. The manner the ecosystem copes with the initial extinction event depends on the degree of competition among the species as well as on the strength of that event. For instance, in the regime of high competition the ecosystem diversity, given by the fraction of surviving species, is practically insensitive to the strength of the initial extinction provided it is not too large, while in the less competitive regime the diversity decreases linearly with the size of the event. In the case of large extinction events we find that no further biotic extinctions take place and, furthermore, that rare species become very unlikely to be found in the ecosystem at equilibrium. In addition, we show that the reciprocal of the EdwardsAnderson order parameter yields a good measure of the diversity of the model ecosystem.
\end{abstract}

PACS: 87.10+e, 87.90.+y, 89.90+n 


\section{Introduction}

Extinction seems to be the final outcome of the evolution of species. In fact, as species survive about 10 million years in the average, nearly all species that have ever existed are extinct and only a very small fraction of them have left their impressions in the fossil record [1, 2]. The causes of the mass extinction events is currently a matter of dispute as there are two main types of explanations [3]. The more traditional one asserts that extinction is caused by external stresses as, for instance, major climate changes and asteroids impacts. This point of view is supported by some evidences such as the unusual quantity of iridium and other noble metals in the rocks that marked the boundary between the Cretaceous and Tertiary periods, when the era of the dinosaurs was replaced by the era of the mammals 泊. Since iridium is more common in asteroids than in the Earth's crust, this finding can be viewed as evidence for an asteroid impact. The alternative explanation asserts that extinctions are caused by the interactions between the species in the ecosystems. In particular, Paine [5] has shown that species richness sometimes can be increased by the predator-mediated coexistence, and the removal of predators can lead to additional species extinctions. Some recent studies indicate that food webs with many species or high connectivity are more likely to lose species as a consequence of the extinction of a single species when compared with more simple food webs [6, 7]. Although this kind of argument seems well suited to explain the so-called background extinctions, it certainly needs some new ingredients to explain mass extinctions as well. In fact, the missing ingredient seems to be the self-organized criticality concept [8], which in this context is best illustrated by the popular BakSneppen model [9]. According to this model, the fitness of each species is affected by the other species to which it is coupled in the ecosystem so that large events in the evolutionary history may be thought of as large coevolutionary avalanches caused by the intrinsic dynamics of the model. In this model, the distribution of the extinction sizes follows a power law, which is a valid candidate for fitting to the experimental data.

Although we recognize that evolution and hence extinction are, as pictured by the models mentioned above, essentially dynamical phenomena, in this work we study these phenomena within the equilibrium statistical mechanics framework of the random replicator model for species coevolution [10, 11, 12, 13]. Deterministic replicator models are commonly used to describe the evolution of self-reproducing entities in a variety of fields such as game theory, prebiotic evolution and sociobiology [14, 15. The random replicator model introduced by Diederich and Opper [10] attempts to model the uncertainties and the overwhelming complexity of the interspecies interactions in biological ecosystems by assuming that those interactions are random. However, it is also assumed that the dynamics is such that a fitness functional (Lyapunov function) is maximized so that the only stationary states are fixed points. In fact, the existence of such a functional leads to a replicator equation with symmetric interspecies interactions [15] which is a severe assumption from the biological standpoint. It allows, however, full use of tools of the equilibrium 
statistical mechanics to study analytically the average properties of the equilibrium states of this kind of disordered ecosystems.

An interesting result of the random replicator model is that in the equilibrium state a fraction of the species is extinct 10]. The mechanism of extinction is clearly outcompetition and, in the absence of any cooperation pressure, only the pair of species with the largest reinforcing interactions will thrive. In this contribution we study the effects of random elimination of a fixed fraction of the species at the outset of the evolutionary process, giving emphasis to the distribution of the remaining species concentrations in the equilibrium state. There are several interesting issues that can be addressed in this framework. For instance, what is the equilibrium situation when the fraction of species eliminated at the beginning is already larger than the fraction that would be extinct naturally due to outcompetition? Furthermore, how does the ecosystem cope with large initial extinction events? In this paper we give clear-cut analytic answers to these questions which are partly corroborated by numerical simulations of the model ecosystem.

The remainder of the paper is organized as follows. In Sec. 2 we introduce the model and discuss the ecological interpretation of the control parameters. The equilibrium properties of the model are derived within the replica-symmetric framework in Sec. 3 and the use of the reciprocal of the Edwards-Anderson order parameter as a measure for the diversity of the ecosystem is suggested. In Sec. A we calculate the distribution of probability of the concentrations of a given species, allowing thus the explicit calculation of the ecosystem diversity as the fraction of surviving species at equilibrium. Finally, in Sec. - we present some concluding remarks.

\section{Model}

We consider an infinite population (ecosystem) composed of individuals belonging to $N$ different species whose fitness $\mathcal{F}_{i}(i=1, \ldots, N)$ are the derivatives $\mathcal{F}_{i}=$ $\partial \mathcal{F} / \partial x_{i}$ of the fitness functional $\mathcal{F}$ defined as

$$
-\mathcal{F}=\mathcal{H}(\mathbf{x})=u \sum_{i} b_{i} x_{i}^{2}+\sum_{i<j} J_{i j} b_{i} x_{i} b_{j} x_{j}
$$

where $x_{i} \in[0, \infty)$ is the fraction of species $i$ and $b_{i}$ is a quenched random variable that takes the values 0 and 1 with probabilities $a$ and $1-a$, respectively. Hence $N a$ randomly chosen species are eliminated at the outset in the average and so henceforth we will refer to $a \in[0,1]$ as the dilution parameter. An effective competition among the species is enforced by requiring that the concentrations of the surviving species satisfy the constraint

$$
\sum_{i=1}^{N} b_{i} x_{i}=Q_{0} N
$$

where $Q_{0}$ is an arbitrary positive constant which gives the scale of the concentrations $x_{i}$. The coupling strengths $J_{i j}$ between species $i$ and $j$ are statistically 
independent quenched random variables with a Gaussian distribution

$$
\mathcal{P}\left(J_{i j}\right)=\sqrt{\frac{N}{2 \pi}} \exp \left[-\frac{\left(J_{i j}\right)^{2} N}{2}\right]
$$

so that $J_{i j}<0$ corresponds to pairs of cooperating species while $J_{i j}>0$ to pairs of competing species. The self-interaction parameter $u \geq 0$ acts as a global cooperation pressure limiting the growth of any single species, and it is crucial to guarantee the existence of a nontrivial thermodynamic limit, $N \rightarrow \infty$. In fact, for large $u$ the minimum of $\mathcal{H}$ corresponds to a homogeneous ecosystem where the surviving species have concentrations $x_{i} / Q_{0}=1 /(1-a) \forall i$. The positive self-interactions means that individuals of a same species compete against themselves, which is quite reasonable as they certainly share the same resources (ecological niche).

The time evolution of the species concentrations is given by the replicator equation

$$
\frac{d x_{i}}{d t}=-x_{i}\left[\frac{\partial \mathcal{H}(\mathbf{x})}{\partial x_{i}}-\frac{1}{N} \sum_{k} x_{k} \frac{\partial \mathcal{H}(\mathbf{x})}{\partial x_{k}}\right] \forall i
$$

which minimizes $\mathcal{H}(\mathbf{x})$ while keeping the term $\sum_{i} b_{i} x_{i}$ constant during the evolution. Hence the fixed points of this equation are the minima of $\mathcal{H}(\mathbf{x})$ and in the following we use the replica formalism to study analytically the statistical properties of these minima.

\section{The replica approach}

Following the standard prescription of performing quenched averages on extensive quantities only [16], we define the average free-energy density $f$ as

$$
-\beta f=\lim _{N \rightarrow \infty} \frac{1}{N}\langle\ln Z\rangle
$$

where

$$
Z=\int_{0}^{\infty} \prod_{i} d x_{i} \delta\left(Q_{0} N-\sum_{i} b_{i} x_{i}\right)\left(Q N-\sum_{i} x_{i}\left(1-b_{i}\right)\right) \mathrm{e}^{-\beta \mathcal{H}(\mathbf{x})}
$$

is the partition function and $\beta=1 / T$ is the inverse temperature. Taking the limit $T \rightarrow 0$ in Eq. (6) ensures that only the states that minimize $\mathcal{H}(\mathbf{x})$ will contribute to $Z$. We impose the additional constraint

$$
\sum_{i} x_{i}\left(1-b_{i}\right)=Q N
$$

to avoid divergences when carrying out the integrals over $x_{i}$. Here $\langle\ldots\rangle$ stands for the average over the coupling strengths $J_{i j}$ as well as over the auxiliary variables 
$b_{i}$. As usual, the evaluation of the quenched average in Eq. (5) can be carried out through the replica method: using the identity

$$
\langle\ln Z\rangle=\lim _{n \rightarrow 0} \frac{1}{n} \ln \left\langle Z^{n}\right\rangle
$$

we first calculate $\left\langle Z^{n}\right\rangle$ for integer $n$, i.e., $Z^{n}=\prod_{\rho=1}^{n} Z^{\rho}$, and then analytically continue to $n=0$. The final result is

$$
\begin{aligned}
-\beta f= & \lim _{n \rightarrow 0} \operatorname{extr} \frac{1}{n}\left\{\sum_{\rho} \hat{p}^{\rho} p^{\rho}-\frac{\beta u}{2} \sum_{\rho} p^{\rho}+\frac{\beta^{2}}{4} \sum_{\rho}\left(p^{\rho}\right)^{2}+\sum_{\rho<\delta} \hat{q}^{\rho \delta} q^{\rho \delta}+\sum_{\rho} Q \hat{Q}^{\rho}\right. \\
& \left.+\frac{\beta^{2}}{2} \sum_{\rho<\delta}\left(q^{\rho \delta}\right)^{2}+\sum_{\rho} Q_{0} \hat{R}^{\rho}+\sum_{b=0}^{1} P_{b} \ln G_{0}\left(b, \hat{p}^{\rho}, \hat{q}^{\rho \delta}, \hat{R}^{\rho}, \hat{Q}^{\rho}\right)\right\}
\end{aligned}
$$

where $P_{0}=a, P_{1}=1-a$, and

$$
\begin{aligned}
G_{0}= & \int_{0}^{\infty} \prod_{\rho} d x^{\rho} \exp \left\{-b \sum_{\rho} \hat{p}^{\rho}\left(x^{\rho}\right)^{2}-b \sum_{\rho<\delta} \hat{q}^{\rho \delta} x^{\rho} x^{\delta}\right. \\
& \left.-b \sum_{\rho} \hat{R}^{\rho} x^{\rho}-(1-b) \sum_{\rho} \hat{Q}^{\rho} x^{\rho}\right\} .
\end{aligned}
$$

We note that while we have calculated the average over the couplings $J_{i j}$ explicitly, we have used the self-averaging property $\frac{1}{N} \sum_{i} \ln G_{0}\left(b_{i}\right)=\sum_{b} P_{b} \ln G_{0}(b)$ to eliminate the site dependence of the $b_{i}$ variables. The relevant physical order parameters are

$$
\begin{aligned}
q^{\rho \delta} & =\frac{1}{N} \sum_{i}\left\langle\left\langle x_{i}^{\rho} x_{i}^{\delta}\right\rangle_{T}\right\rangle \quad \rho<\delta \\
p^{\rho} & =\frac{1}{N} \sum_{i}\left\langle\left\langle\left(x_{i}^{\rho}\right)^{2}\right\rangle_{T}\right\rangle
\end{aligned}
$$

which measure the overlap between a pair of different equilibrium states $\mathbf{x}^{\rho}$ and $\mathbf{x}^{\delta}$, and the overlap of an equilibrium state $\mathbf{x}^{\rho}$ with itself, respectively. Here, $\langle\ldots\rangle_{T}$ stands for a thermal average taken with the Gibbs probability distribution

$$
\mathcal{W}(\mathbf{x})=\frac{1}{Z} \delta\left(Q_{0} N-\sum_{i} x_{i}\right) \delta\left(Q N-\sum_{i}\left(1-b_{i}\right) x_{i}\right) \exp [-\beta \mathcal{H}(\mathbf{x})]
$$

To proceed further we assume that the saddle-point parameters are symmetric under permutations of the replica indices, i.e., $p^{\rho}=p, \hat{p}^{\rho}=\hat{p}, q^{\rho \delta}=q, \hat{q}^{\rho \delta}=$ $\hat{q}, \hat{R}^{\rho}=\hat{R}$ and $\hat{Q}^{\rho}=\hat{Q}$. With this prescription the evaluation of Eq. (9) is straightforward yielding the following replica-symmetric free energy density

$$
-\beta f=-\frac{\beta q y}{2}-\beta Q_{0} \hat{R}+a+a \ln \left(\frac{Q}{a}\right)+\frac{1-a}{2} \ln \left(\frac{\pi}{2 \beta(2 u-y)}\right)
$$




$$
+\beta(1-a) \frac{\hat{R}^{2}+q}{2(2 u-y)}+(1-a) \int_{-\infty}^{\infty} D z \ln \operatorname{erfc}\left[\frac{\sqrt{\beta}(\hat{R}+z \sqrt{q})}{\sqrt{2(2 u-y)}}\right]
$$

where $y=\beta(p-q)$ and $D z=d z \exp \left(-z^{2} / 2\right) / \sqrt{2 \pi}$ is the Gaussian measure. Already at this stage we can see that the concentration of species eliminated at the outset, given by the parameter $Q$, decouples from the other physical parameters and hence does not have any effect upon them. In the zero-temperature limit the saddle-point equations $\partial f / \partial q=0, \partial f / \partial y=0$ and $\partial f / \partial \hat{R}=0$ are given by

$$
\begin{gathered}
\Delta=2 \frac{\sqrt{q}}{Q_{0}}(u-y), \\
2 y(2 u-y)=(1-a) \operatorname{erfc}(-\Delta / \sqrt{2}),
\end{gathered}
$$

and

$$
\frac{(1-a) \Delta}{\sqrt{2 \pi}} \exp \left(-\Delta^{2} / 2\right)=(2 u-y)^{2}-(2 u-y)\left(\Delta^{2}+1\right) y .
$$

We note that the parameter associated to the concentration of surviving species $Q_{0}$ appears only as a scale of $q$ and so henceforth we will set $Q_{0}=1$ without loss of generality. In the replica-symmetric framework the Edwards-Anderson order parameter $q$ is defined by

$$
q=\left\langle\frac{1}{N} \sum_{i}\left\langle x_{i}\right\rangle_{T}^{2}\right\rangle .
$$

If the concentrations $x_{i}$ were normalized to 1 rather than to $N$ then $q$ would give the probability that two randomly selected individuals are of the same species, a quantity known as Simpson's index [17]. Nevertheless, we can still give a simple physical interpretation to $q$. For instance, values of $q$ of order of 1 indicate the coexistence of a macroscopic number of species (i.e., $x_{i} \approx 1$ for an extensive number of species), while large values of $q$ signalize the dominance of a few species only (i.e., $x_{i} \approx N$ for a finite number of species). Of course, this interpretation is equivalent to that given above for Simpson's index, and so we can view $1 / q$ as a measure of the diversity of the ecosystem. In Fig. 11 we present $1 / q$ as a function of the dilution parameter $a$ for several values of the cooperation pressure $u$. The results of the numerical solution of the replicator equation, Eq. (何), for $N=500$ are also presented. Each data point is the average over 100 realizations of the matrix of coupling strengths, starting with an uniform distribution of concentrations. Since the labeling of the species is arbitrary we can set $b_{i}=0$ for $i \leq a N$ and $b_{i}=1$ otherwise, without loss of generality. In addition, we choose $a$ such that $a N$ is integer for simplicity. In agreement with our interpretation, for $a$ close to 1 we can observe the vanishing of $1 / q$, which characterizes an ecosystem composed of a few species only. For small values of $u$ the analytical results show the existence of a maximum of diversity for a nonzero value of the dilution parameter (see the inset in Fig. 1); the numerical results however do not corroborate this finding. This 


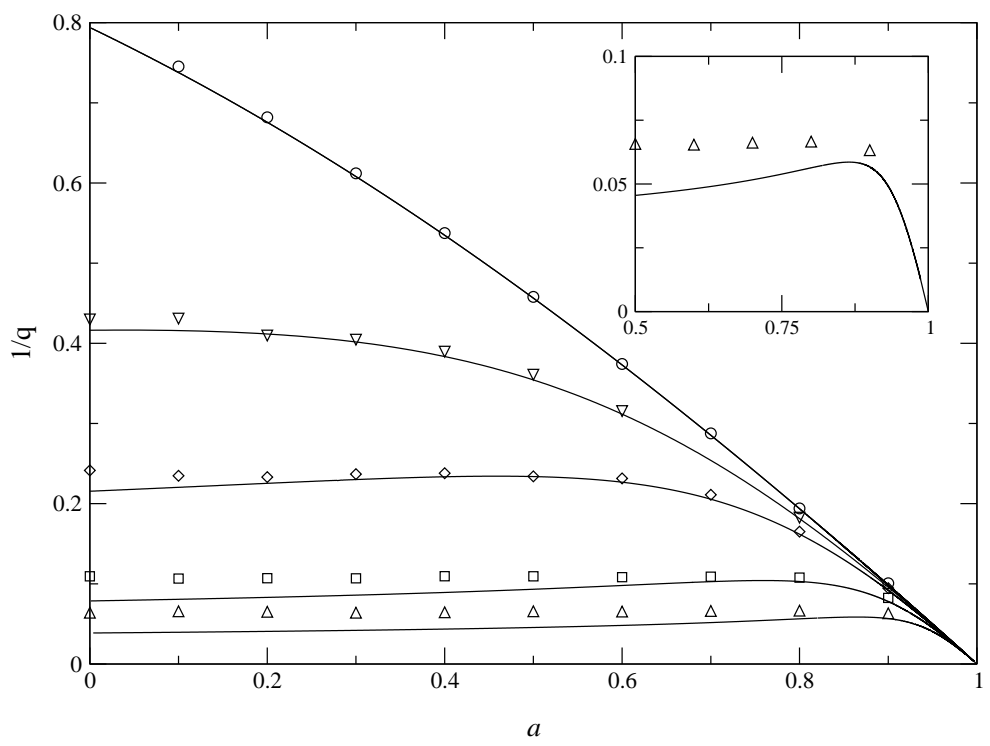

Figure 1: The diversity $1 / q$ as a funcion of the dilution parameter $a$ for (top to bottom) $u=1.3(\bigcirc), 0.8(\nabla), 0.6(\diamond), 0.4(\square)$ and $0.3(\triangle)$. The symbols are the results of the numerical solution of the replicator equation. The inset highlights the region of the diversity maximum.

discrepancy can be explained by the instability of the replica-symmetric solution. In fact, carrying out the standard local stability analysis [18], we find that this solution is locally stable wherever the condition

$$
\lambda=-1+\frac{1}{2(2 u-y)^{2}} \operatorname{erfc}\left(-\frac{\Delta}{\sqrt{2}}\right)<0
$$

is satisfied. Figure 2 shows the regions in the plane $(a, u)$ where the replicasymmetric solution is stable. In particular, we find that for $a=0$ this solution is stable for $u>1 / \sqrt{2}$ while for $a=1$ it is stable for $u>1 / 2$. Hence, the maxima observed in Fig. 11 are indeed artifacts of the replica-symmetry framework. Nevertheless, the agreement between the analytical and numerical results is already excelent for $u>0.6$. The rather puzzling independence of the diversity on the dilution parameter for small $u$ has a simple explanation as will be seen in the next section.

\section{Discussion}

Although the interpretation of the reciprocal of the Edwards-Anderson order parameter as the ecosystem diversity yields some information on the distribution of species at equilibrium, a better understanding is achieved by calculating explicitly 


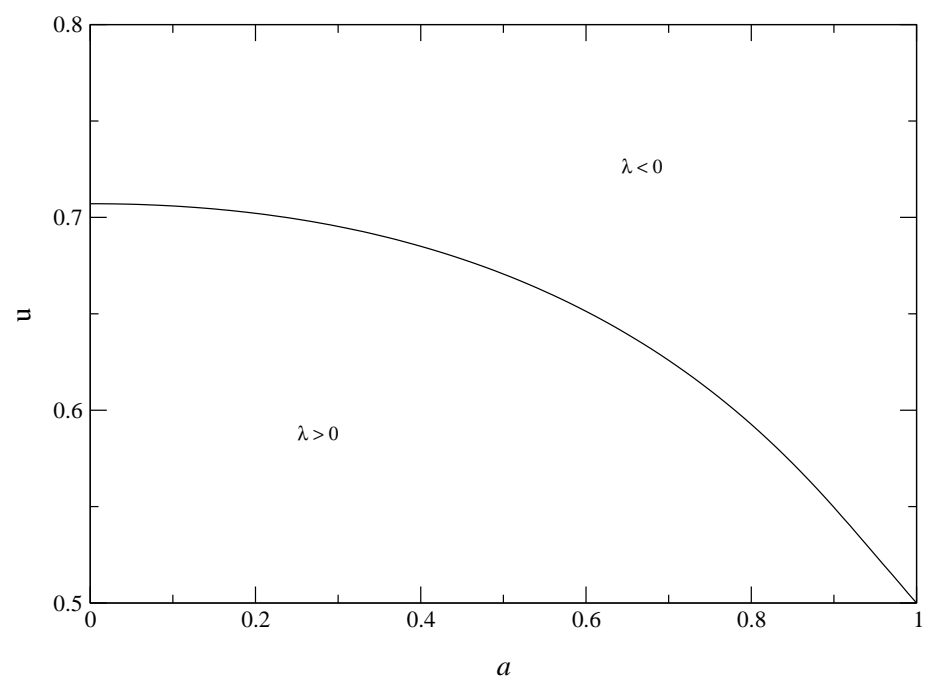

Figure 2: Almeida-Thouless line separating the regions of stability $(\lambda<0)$ and instability $(\lambda>0)$ of the replica-symmetric solution.

the probability distribution that the concentration of one of the $(1-a) N$ remaining species, say $x_{k}$, assumes the value $x$, defined by

$$
\mathcal{P}_{k}(x)=\lim _{\beta \rightarrow \infty}\left\langle\int_{0}^{\infty} \prod_{j} d x_{j} b_{k} \delta\left(x_{k}-x\right) \mathcal{W}(\mathbf{x})\right\rangle
$$

with $\mathcal{W}(\mathbf{x})$ given by Eq. (13). As all non-vanishing species concentrations are equivalent we can write $\mathcal{P}_{k}(x)=\mathcal{P}(x) \forall k$. Hence to evaluate Eq. (20) we introduce the auxiliary energy

$$
\mathcal{H}_{\text {aux }}(\mathbf{x})=\mathcal{H}(\mathbf{x})+h \sum_{k} b_{k} \delta\left(x_{k}-x\right),
$$

so that

$$
\mathcal{P}(x)=-\left.\lim _{\beta \rightarrow \infty} \frac{1}{N \beta} \frac{\partial\left\langle\ln Z_{a u x}\right\rangle}{\partial h}\right|_{h=0}
$$

where $Z_{\text {aux }}$ is the partition function (6) with $\mathcal{H}$ replaced by $\mathcal{H}_{\text {aux }}$. Using Eq. (22) the calculations needed to evaluate $\mathcal{P}(x)$ become analogous to those used in the evaluation of the free-energy density (14). In addition, to handle a possible singularity in the limit $\beta \rightarrow \infty$ it is more convenient to deal with the cumulative distribution function $\mathcal{C}(x)=\int_{0}^{x} d x^{\prime} \mathcal{P}\left(x^{\prime}\right)$. Carrying out the calculations within the replica-symmetric framework we obtain

$$
\mathcal{C}(x)=(1-a)\left\{1-\frac{1}{2} \operatorname{erfc}\left[\frac{1}{\sqrt{2}}\left(\frac{x(2 u-y)}{\sqrt{q}}-\Delta\right)\right]\right\}
$$


where $q, y$ and $\Delta$ are given by the saddle-point equations (15)-(17). In Fig. 3 we show $\mathcal{C}(x)$ for $u=0.8$ and several values of $a$. The first point to note is that $\lim _{x \rightarrow \infty} \mathcal{C}(x)=1-a$ yields the fraction of surviving species at the outset, as expected. In addition, a nonzero value of $\mathcal{C}(0)$ indicates that the probability distribution $\mathcal{P}(x)$ has a delta peak at $x=0$ and so $\mathcal{C}(0)$ actually yields the fraction of the species that survived the initial externally induced extinction event but that were extinct later on due to outcompetition. In the regime of large dilution, say $a>0.8$ in Fig. 3, the cumulative distribution is very small and practically constant for small concentrations, indicating that no further extinctions have taken place and, furthermore, that rare species are very unlikely to be found in the ecosystem at equilibrium. We note that the numerical simulations yield results practically indistinguishable from the analytical ones. The rough independence of the diversity $1 / q$ on the dilution parameter $a$ observed in Fig. 1 for small $u$ is easily understood with the aid of the cumulated distributions. In fact, a direct measure of the ecosystem diversity is given by the fraction of surviving species $1-a-\mathcal{C}(0)$, which is shown in Fig. 4 as function of $a$. (We recall that $a$ is the fraction of species that were extinct at the outset due to some external stress and $\mathcal{C}(0)$ is the fraction that died out due to outcompetition.) The remarkable similarity between these figures corroborates our interpretation of $1 / q$ as a measure of the diversity. Clearly, the diversity is insensitive to variations of $a$ whenever the fraction of extinct species in the undisturbed ecosystem (i.e. $\mathcal{C}(0)$ calculated at $a=0$ ) is already considerably larger than $a$, so that the species eliminated at the outset would probably be extinct later on anyway.

\section{Conclusion}

Although the dynamics of the random replicator model may not look very appealing, in the sense that it always leads to fixed points, the frustration caused by the competition between the concentration limiting self-interactions $(u>0)$ and the tendency to unlimited growth of pairs of strongly cooperative $\left(J_{i j}<0\right)$ species results in a highly nontrivial equilibrium, characterized by many meta-stable states [10] and a phase of replica symmetry breaking [11]. Of course, these very features make some aspects of the dynamics (e.g., slow relaxation and hysteresis effects) nontrivial as well. The wealth of ecologically relevant issues that can be addressed within this equilibrium framework can be appreciated, for instance, in the case of high-order interactions among the species where it has been reported the emergence of a threshold value which gives a lower bound to the concentration of the surviving species, preventing then the existence of rare (low concentration) species in the ecosystem 13.

An important outcome of the equilibrium analysis of the random replicator model is the finding that in order to reduce the degree of frustration a fraction of the species dies out [10]. This type of extinction has clearly a biotic cause, namely, outcompetition 19. In this paper we study how the model ecosystem 


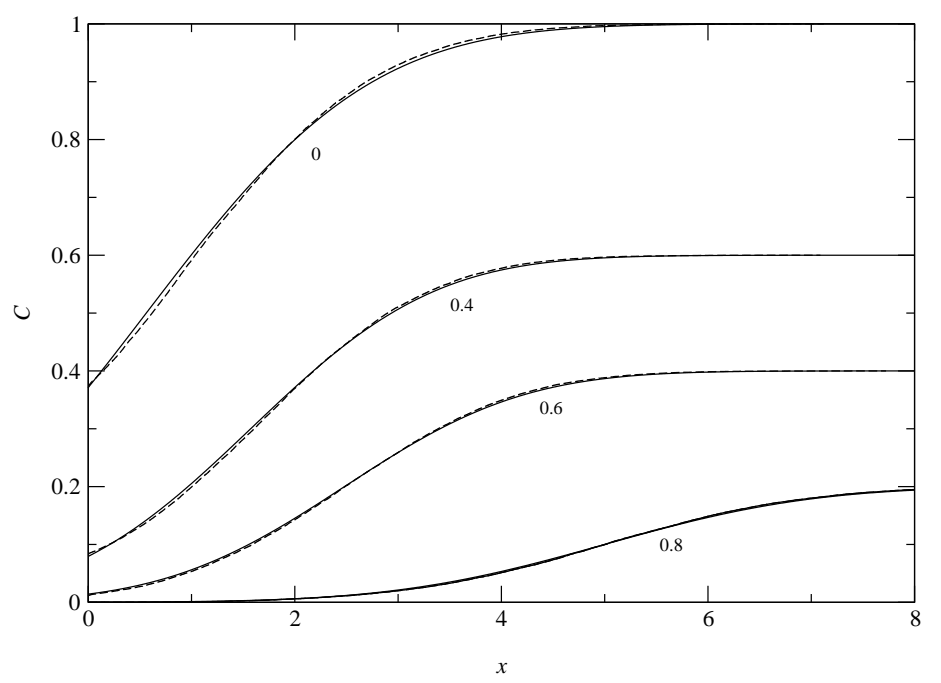

Figure 3: Cumulative distribution of the concentration of the initially surviving species in equilibrium for $u=0.8$ and (top to bottom) $a=0,0.4$, 0.6 , and 0.8. The dashed curves are the results of the numerical solution of the replicator equation.

copes with abiotic or externally induced extinction, in which a fraction of randomly chosen species is eliminated at the beginning of the coevolutionary process. We find that in the regime of high competition (small $u$ ) the ecosystem diversity, i.e., the fraction of surviving species is practically insensitive to the strength $a$ of the initial extinction provided it is not too large, while in the less competitive regime (large $u$ ) the diversity decreases linearly with increasing $a$. In the case of a large extinction event we find that no further (biotic) extinctions take place and, furthermore, that rare species become very unlikely to be found in the ecosystem at equilibrium. This is distinct from the result mentioned above for the case of high-order interactions where the probability of finding rare species in the ecosystem is strictly null [13].

An interesting by-product of our investigation is the finding that the reciprocal of the Edwards-Anderson order parameter (i.e., the replica-symmetric overlap between two equilibrium states) serves as an easy-to-calculate measure of the diversity of the model ecosystem. This opens the exciting possibility of interpreting the different hierarquical levels of the overlap order parameter in the full replica symmetry breaking scheme [16] as different levels of a phylogenetic tree that gives the relations of dependence (viewed as ancestrality) among the species.

\section{Acknowledgments}

The work of J.F.F. is supported in part by Conselho Nacional de Desenvolvi 


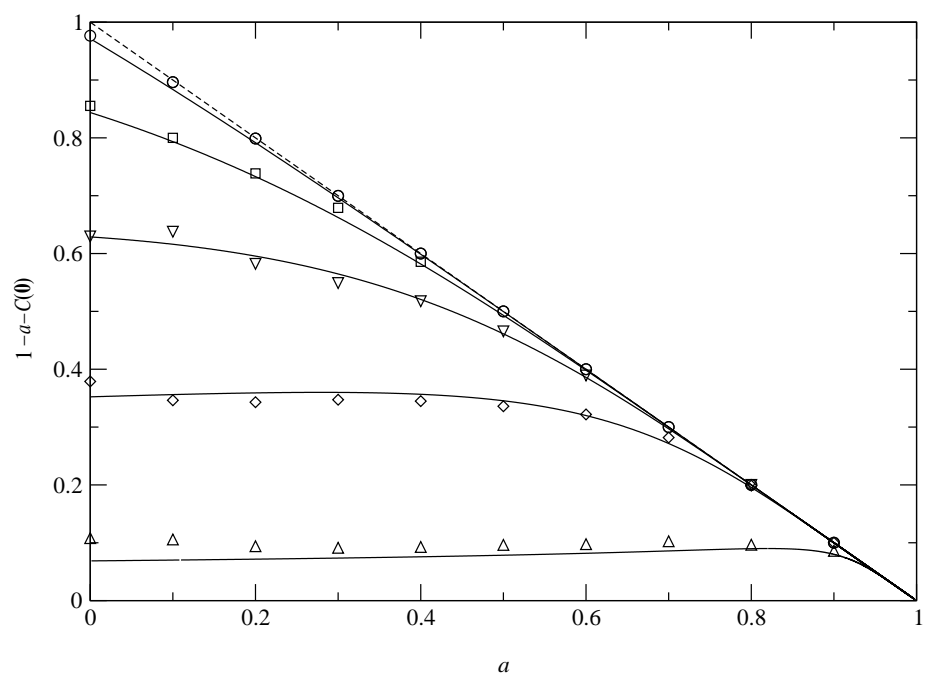

Figure 4: Fraction of surviving species as a function of the dilution parameter $a$ for (top to bottom) $u=1.3(\bigcirc), 1.0(\square), 0.8(\nabla), 0.6(\diamond)$ and $0.3(\triangle)$. The symbols are the results of the numerical solution of the replicator equation. The dashed line is the fraction of species at the beginning of the coevolutionary process. 
mento Científico e Tecnológico $(\mathrm{CNPq})$ and Fundação de Amparo à Pesquisa do Estado de São Paulo (FAPESP), Proj. No. 99/09644-9. V.M.O. is supported by FAPESP.

\section{References}

[1] D. Raup and J. J. Sepkoski Jr, Proc. Natl. Acad. Sci. U.S.A. 81, 801 (1984).

[2] M. E. J. Newman, adap-org/9910003, 1999

[3] M. E. J. Newman and R. G. Palmer, adap-org/9908002, 1999

[4] L. W. Alvarez, W. Alvarez, F. Asaro and H. V. Michel, Science 208, 1095 (1980).

[5] R. T. Paine, Am. Nat. 100, 65 (1966).

[6] S. L. Pimm, Oikos 33, 351 (1979).

[7] S. L. Pimm, Oikos 35, 139 (1980).

[8] P. Bak, C. Tang and K. Wiesenfeld, Phys. Rev. Lett. 59, 381 (1987).

[9] P. Bak and K. Sneppen, Phys. Rev. Lett. 71, 4083 (1993).

[10] S. Diederich and M. Opper, Phys. Rev. A 39, 4333 (1989).

[11] P. Biscari and G. Parisi, J. Phys. A 28, 4697 (1995).

[12] M. Opper and S. Diederich, Comp. Phys. Comm. 121, 141 (1999).

[13] V. M. de Oliveira and J. F. Fontanari, Phys. Rev. Lett 85, 4984 (2000).

[14] P. Schuster and K. Sigmund, J. Theor. Biol 100, 533 (1983).

[15] J. Hofbauer and K. Sigmund, Dynamical Systems and the Theory of Evolution (Cambridge Univ. Press, Cambridge UK, 1988).

[16] M. Mézard, G. Parisi and M. A. Virasoro, Spin Glass Theory and Beyond (World Scientific, Singapore, 1987).

[17] E. H. Simpson, Nature 163, 688 (1949).

[18] J. R. L. Almeida and D. J. Thouless, J. Phys. A 11, 983 (1978).

[19] J. Maynard Smith, Phil. Trans. R. Soc. London B 325, 241 (1989) 